\title{
High S phase kinase-associated protein 2 expression is a potential prognostic biomarker for glioma
}

\author{
ZHI-JIAN CHENG ${ }^{1,2 *}$, HONG-QING CAI ${ }^{3 *}$, MIN-JIE ZHANG $^{1,2}$, YI ZHONG $^{1,2}$, JIE HE $^{1}$, \\ QING YUAN $^{1,2}$, JIA-JIE HAO ${ }^{2}$, MING-RONG WANG ${ }^{2}$ and JING-HAI WAN ${ }^{1}$
}

\author{
${ }^{1}$ Department of Neurosurgery, The Second Affiliated Hospital of Anhui Medical University, Hefei, Anhui 230601; \\ Departments of ${ }^{2}$ State Key Laboratory of Molecular Oncology and ${ }^{3}$ Neurosurgery, National Cancer Center/Cancer Hospital, \\ Chinese Academy of Medical Sciences and Peking Union Medical College, Beijing 100021, P.R. China
}

Received July 28, 2019; Accepted February 7, 2020

DOI: $10.3892 / 01.2020 .11818$

\begin{abstract}
S phase kinase-associated protein 2 (SKP2), a substrate recognizing protein, serves an important role in promoting cell cycle progression through ubiquitination and degradation of cell cycle inhibitors. In the present study, the clinical significance of SKP2 in gliomas was studied; 395 glioma specimens and 20 non-neoplastic tissues were collected and immunohistochemical analysis was performed. $\chi^{2}$ test was used to assess the associations between SKP2 expression and clinical parameters. Overall survival (OS) curves were plotted according to the Kaplan-Meier method. In the tested glioma samples, SKP2 expression was mainly observed in glioblastomas (GBMs). Survival analysis demonstrated that the overall survival time of the high SKP2 expression group was lower compared with the low SKP2 expression group (median OS, 10.04 months vs. 16.50 months; $\mathrm{P}=0.003$ ). Moreover, SKP2 was independently associated with an unfavorable prognosis in GBMs. In addition, the expression of SKP2 was associated with the expression of phosphorylated retinoblastoma protein and the epidermal growth factor receptor. A combination of SKP2 expression along with isocitrate dehydrogenase 1 (IDH1) mutations and telomerase reverse transcriptase (TERT) promoter mutations was used to classify glioma patients for survival analysis. Patients with low SKP2 expression, IDH1
\end{abstract}

Correspondence to: Dr Jing-Hai Wan, Department of Neurosurgery, The Second Affiliated Hospital of Anhui Medical University, 678 Fu Rong Road, Hefei, Anhui 230601, P.R. China

E-mail: wanjinghai@sina.com

Dr Hong-Qing Cai, Department of Neurosurgery, National Cancer Center/Cancer Hospital, Chinese Academy of Medical Sciences and Peking Union Medical College, 17 Pan Jia Yuan North Road, Beijing 100021, P.R. China

E-mail: phonecy@126.com

*Contributed equally

Key words: glioma, S phase kinase-associated protein 2, immunohistochemistry, biomarker, prognosis mutation and wild-type TERT promoter demonstrated the longest survival time. The findings of the present study, indicate that SKP2 is a potential prognostic biomarker in patients with GBMs.

\section{Introduction}

Glioma is the most common primary malignant brain tumor in 2016 worldwide (1), which can be classified into grades I-IV according to the World Health Organization (WHO) 2016 classification system (2). Glioblastoma multiforme (GBM) is also known as high-grade glioma (HGG), which is the most aggressive form of glioma, whereas the others are considered low-grade gliomas (LGG) (3). Although, significant efforts have been made to find effective treatment approaches for glioma, the 5 year survival rate of GBM remains poor $(\sim 5 \%)$ (1). Studies investigating molecular biomarkers have demonstrated progress and provided optimism for the future, compared with the limited success of conventional therapies (4). Thus, there is an urgent need for the development of novel and effective biomarkers for molecular subtyping, predicting prognosis and the design of personalized treatments for patients with glioma.

F-box proteins are the substrate recognizing subunits of Skp1-Cullin 1-F-box ubiquitin ligase complex, which recognizes specific targets and promotes their ubiquitination $(5,6)$. $\mathrm{S}$ phase kinase-associated protein 2 (SKP2) is a typical F-box protein that serves a crucial role in multiple cellular processes including proliferation, invasion and metastasis (7). It functions as a promoter of cell cycle progression via the ubiquitination and subsequent degradation of cell cycle inhibitors, including p21, p27, p57 and cyclin E (8). As the majority of SKP2 substrates are tumor suppressors, it is widely considered to be an oncoprotein (9). Previous studies have indicated that SKP2 is upregulated in a variety of human malignancies, including breast cancer and gastric cancer, and is associated with carcinogenesis and tumor progression $(10,11)$. Emerging evidence indicates that SKP2 is also involved in chemoresistance and may be a novel therapeutic target. For example, the inhibition of SKP2 sensitizes lung cancer cells to paclitaxel or rapamycin $(12,13)$. In gliomas, several in vitro experiments have demonstrated that suppressing the expression of SKP2 significantly blocks cell cycle progression, induces 
cell apoptosis and inhibits cell invasion $(14,15)$. However, the clinical implications of SKP2 in gliomas remain unclear, despite SKP2 detection being reported in certain operative specimens $(16,17)$.

Isocitrate dehydrogenase 1 (IDH1) and telomerase reverse transcriptase promoter (TERTp) mutation have been used in the clinic for glioma (18). IDH1 mutation was an early event in glioma development, which was observed in almost $80 \%$ of grade II-III gliomas and secondary GBM (19). TERTp mutation is inversely correlated with IDH1 mutation in glioblastoma, and is therefore predictive of poor prognosis (20). Phosphorylated retinoblastoma protein ( $\mathrm{p}-\mathrm{Rb})$ is also known as the cell cycle regulating protein $(21)$. $\mathrm{Rb}$ protein binds and inhibits the transcription factor for the E2F family, which causes cell cycle arrest at $\mathrm{G}_{1}(22)$. When $\mathrm{Rb}$ is phosphorylated, it dissociates from $\mathrm{E} 2 \mathrm{~F}$ and allows progression to the $\mathrm{S}$ phase (22). Epidermal growth factor receptor (EGFR) is a member of the transmembrane tyrosine kinases and it overexpressed in $60 \%$ of glioblastoma (23). Furthermore, inhibiting the expression of $\mathrm{p}-\mathrm{Rb}$ and EGFR can result in tumor cell arrest in the $\mathrm{G}_{1}$ phase and dysregulate the expression of SKP2 $(24,25)$.

The aim of the present study was to investigate the expression and prognostic value of SKP2 in samples from 395 patients with glioma. The results of the present study demonstrated that SKP2 is mainly expressed in glioblastomas (GBMs) and high expression of SKP2 indicates poor survival. The findings of the present study demonstrate that SKP2 is a novel prognostic biomarker in GBMs.

\section{Materials and methods}

Patients and collection of clinical samples. A total of 395 specimens were obtained from patients with glioma who were enrolled in the present study. Of these, 36 were astrocytomas, 21 oligodendrogliomas, 40 anaplastic astrocytomas, 24 anaplastic oligodendrogliomas, 269 GBMs and 5 were other types. There were 234 male and 161 female patients. The mean age was 48 years, ranging from 5-79 years. All patients underwent tumor resection between September 2011 and January 2018 in the Department of Neurosurgery at the National Cancer Center (NCC)/Cancer Hospital of Chinese Academy of Medical Sciences (Beijing, China). None of the patients had a history of other tumors and the neurosurgical treatment was performed to treat glioma alone. All the samples used were residual specimens collected for diagnosis. A total of 20 non-neoplastic tissues were acquired using two methods: Normal brain tissues around tumor and edema tissues surrounding the high-grade gliomas. None of the patients received neoadjuvant treatment prior to surgery and they all signed separate informed consent forms for the sample collection and molecular analyses. Data were recorded for the clinical/pathological parameters of tumors; including age, sex of patients and the WHO grade of tumors. Follow-up was performed every 3 months and 25 patients dropped out prior to the last follow-up call. The present study was approved by the Ethics Committee of the NCC (approval no. NCC2014G-12; Beijing, China).

Tissue microarray and immunohistochemistry. Tumor samples were fixed in $10 \%$ formalin for $24 \mathrm{~h}$ at room temperature following resection, and embedded in paraffin. Tissue microarrays were prepared as previously described (26). A total of three tissue cores were selected from every primary paraffin-embedded samples $(n=359)$. The microarrays were cut into $4 \mu \mathrm{m}$-thick sections. As it is difficult to acquire normal brain tissues, normal tissues $(n=20)$ from patients with glioma were used as controls in the current study. The sections were baked at $72^{\circ} \mathrm{C}$ for $1 \mathrm{~h}$, then deparaffinized with xylene and rehydrated in gradient ethanol including 100, 85 and $75 \%$ for $15 \mathrm{~min}$. Citrate buffer (ZSGB-BIO, Ltd.) was diluted to $\mathrm{pH} 6.0$ and heated to boil for antigen retrieval. Subsequently, specimens were kept in $95-98^{\circ} \mathrm{C}$ citrate buffer by microwaving for $20 \mathrm{~min}$. Then, endogenous peroxidase activity was blocked with $3 \%$ hydrogen peroxide at room temperature for $10 \mathrm{~min}$. The primary SKP2 antibody (1:100; cat. no. 2652S; CST Biological Reagents Co., Ltd.), anti-isocitrate dehydrogenase 1 (IDH1 ${ }^{\mathrm{R} 132 \mathrm{H}}$ ) antibody (working solution; cat. no. ZM0447; OriGene Technologies, Inc.), phosphorylated retinoblastoma protein (p-Rb) antibody (1:100; cat. no. 8516T, CST Biological Reagents Co., Ltd.), epidermal growth factor receptor (EGFR) antibody (working solution; cat. no. ZA-0505; OriGene Technologies, Inc.) were added and the sections were incubated at $4^{\circ} \mathrm{C}$ overnight. Following, 4 washes with PBS, the PV-9000 kit (working solution; cat. no. PV-9000; OriGene Technologies, Inc.) was used for color development according to the manufacturer's protocols. Next, DAB and hematoxylin were used for counterstaining at room temperature for $3 \mathrm{~min}$ and $8 \mathrm{sec}$, respectively. Finally, the slides were dehydrated and mounted with coverslips. Slides were scanned using a NanoZoomer (Japan SLC, Inc.) high-resolution scanner with maximum magnification, $x 400$. NDP analysis software (Visiopharm) was used to identify immunostaining and calculate the proportion of positive cells. The cutoff values were $10 \%$ for IDH $1^{\mathrm{R} 132 \mathrm{H}}$ and $\mathrm{p}-\mathrm{Rb}$. The immune scores of EGFR protein expression were evaluated as described previously (27).

DNA extraction and sanger sequencing. Genomic DNA was extracted from consecutive formalin fixation and paraffin embedding glioma sections of $10 \mu \mathrm{m}$ using the QIAmp DNA mini kit (Qiagen $\mathrm{GmbH}$ ). The telomerase reverse transcriptase (TERT) promoter region was amplified with the forward primer, 5'-TTCCAGCTCCGCCTCCT-3' and the reverse primer, 5'-GCGCTGCCTGAAACTCG-3'. PCR was performed with an initial denaturing step at $95^{\circ} \mathrm{C}$ for $5 \mathrm{~min}$; then 30 cycles of $96^{\circ} \mathrm{C}$ for $15 \mathrm{sec}, 60^{\circ} \mathrm{C}$ for $30 \mathrm{sec}$ and $72^{\circ} \mathrm{C}$ for $30 \mathrm{sec}$; followed by a final extension at $72^{\circ} \mathrm{C}$ for $10 \mathrm{~min}$. The PCR products were sequenced by Beijing Tianyi Huiyuan Bioscience and Technology Inc.

Statistical analysis. Significant differences between two groups were determined by the Mann-Whitney U test. X-tile software v3.6.1 (Rimm Lab, Yale School of Medicine) was used to ascertain the optimal cut-off points for survival analysis. Data were represented as percentages. There were no biological replicates. The $\chi^{2}$ test was used to assess the associations between SKP2 expression and clinicopathological parameters. Overall survival (OS) curves were plotted according to the Kaplan-Meier method, with the log-rank test applied for comparison. Multiple Cox regression analysis was used to predict independent prognostic factors. All statistical analyses 

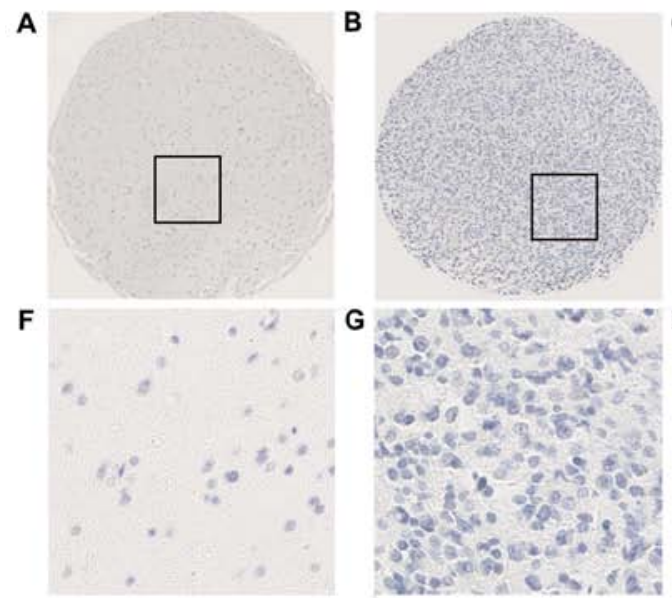
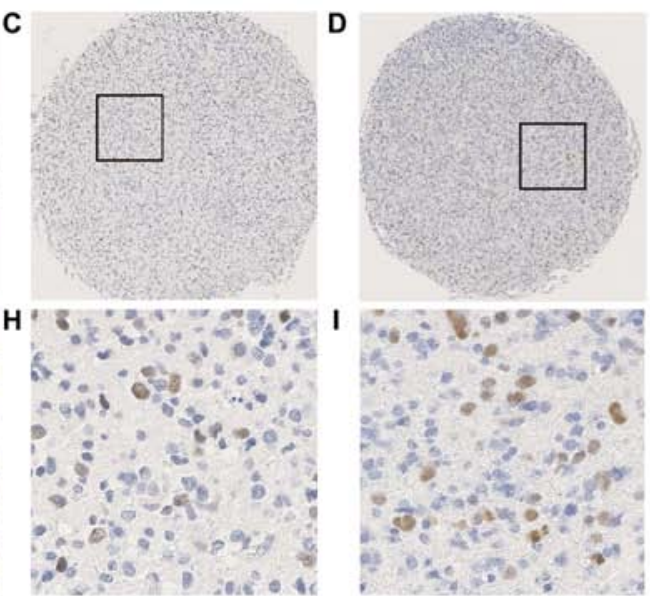

E
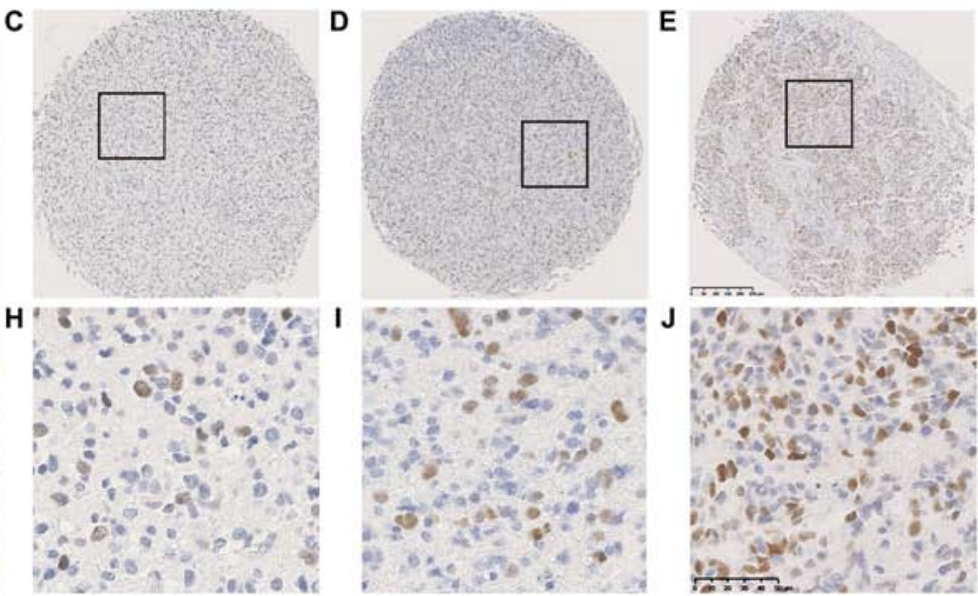

Figure 1. Representative immunostaining images of $\mathrm{S}$ phase kinase-associated protein 2 protein expression. Blue and brown staining represents negative and positive expression of SKP2, respectively. (A and F) Non-neoplastic brain tissues, negative expression. (B and G) Glioma specimens, negative expression; $(\mathrm{C}$ and $\mathrm{H}$ ) low expression; (D and I) moderate expression; and (E and J) high expression. Framed portions in low-resolution images (magnification x100) are presented in corresponding high-resolution images (magnification $\mathrm{x} 400$ ).

were performed using both SPSS Statistics v21.0 (IBM Corp.) and GraphPad Prism v5.0 (GraphPad Software, Inc.). $\mathrm{P}<0.05$ was considered to indicate a statistically significant difference. All the tests performed were two-sided.

\section{Results}

Association between SKP2 expression and clinicopathological parameters in glioma. SKP2 expression was investigated using immunohistochemistry in a total of 395 glioma tissues. These specimens included grade II (60), III (66) and IV (269) tumors (Table I). A total of 20 paired non-neoplastic brain tissues were used as the control. Positive immunostaining of SKP2 was observed in the nuclei of glioma cells, whereas no SKP2 signal was presented in non-neoplastic tissues (Fig. 1). Glioma tissues with different degrees of immunostaining for SKP2, including negative, low, moderate and high expression (Fig. 1). SKP2 expression was detected in $18.0 \%$ (71/395) of all glioma tumors, with $0.0 \%(0 / 60)$ in grade II gliomas, $9.1 \%(6 / 66)$ in grade III gliomas and 24.2\% (65/269) in glioblastomas (Fig. 2). SKP2 expression was significantly associated with tumor grade and histology $(\mathrm{P}<0.001$; Table I). High SKP2 expression levels were more frequently observed in GBM tissues (Table I). However, SKP2 expression was not associated with sex, age and Karnofsky Performance Status (KPS) (Table I).

SKP-2 expression is associated with $p-R b$ and EGFR expression, but not with the TERT promoter mutation. Expression of $\mathrm{p}-\mathrm{Rb}$, EGFR and a mutation in the TERT promoter were found in patients with glioma. Positive immunostaining of $\mathrm{p}-\mathrm{Rb}$ and EGFR was in the nuclei and cytoplasm, respectively (Fig. 3A and C). The TERT promoter mutation was observed in $50.5 \%$ of patients with glioma. The exact mutation is presented in Fig. S1. Linear correlation analysis showed that SKP2 expression was significantly correlated with $\mathrm{p}-\mathrm{Rb}$ expression $(\mathrm{P}<0.001$ in all gliomas; Fig. 3B). Mann-Whitney $\mathrm{U}$ test revealed that SKP2 expression was strongly associated with EGFR expression $(\mathrm{P}<0.001$ in total group; $\mathrm{P}=0.006$ in HGG and $\mathrm{P}=0.005$ in LGG; Fig. 3D). No correlation was observed between SKP2 expression and the TERT promoter mutation $(\mathrm{P}>0.05$, data not shown).

SKP2 expression is associated with a poor prognosis in patients with glioma. Using X-tile software, the optimum cut-off value for SKP2 expression was defined as 10\%. Subsequently, univariate analysis was performed to examine the prognostic significance in LGG and HGG. It revealed that elevated SKP2 expression was associated with a poor prognosis in patients with glioma compared with low expression [hazard ratio (HR), 4.2; LGG median survival times, 67.0 months vs. 11.9 months; $\mathrm{P}<0.001$; Fig. 4A], (HR, 1.7; HGG median survival times, 16.5 months vs. 10.4 months; $\mathrm{P}=0.003$; Fig. 4B). In HGG, patients with radiotherapy (median survival times, 22.3 months vs. 19.6 months; $\mathrm{P}<0.001$; Fig. $4 \mathrm{C}$ ) or chemotherapy (median survival times, 19.3 months vs. 12.8 months; $\mathrm{P}<0.001$; Fig. 4D) showed longer survival times. The associations between SKP2 expression and sensitivity to adjuvant treatments were also investigated. The results demonstrated that in HGG patients who received radiotherapy or chemotherapy, patients with a low SKP2 expression had a longer survival time (radiotherapy median OS time, 21.0 months vs. 9.0 months; $\mathrm{P}=0.012$; Fig. 4E; chemotherapy median OS time, 18.5 months vs. 7.0 months; $\mathrm{P}=0.012$; Fig. 4F).

Prognostic value of SKP2 in patients with glioma. To further validate the prognostic value of SKP2, a multiple Cox proportional hazards regression analysis was performed using clinical and genetic variables, including sex, age, KPS, adjuvant treatments, TERT promoter mutation, IDH1 mutation and SKP2 expression in HGG (Table II). Both univariate and multivariate analyses revealed that KPS, adjuvant treatments, IDH1 mutation and SKP2 expression, but not sex, age and TERT promoter mutation were associated with the OS time of the patients (Table II).

Using SKP2, IDH1 mutation and TERT promoter mutation data classifies patients with glioma into 3 subgroups. A previous study reported the clinical relevance of IDH1 
Table I. Clinical and pathological characteristics of patients with glioma $(n=395)$.

\begin{tabular}{|c|c|c|c|c|}
\hline \multirow[b]{2}{*}{ Variable } & \multirow[b]{2}{*}{ Patients, $\mathrm{n}$} & \multicolumn{2}{|c|}{ SKP2 expression } & \multirow[b]{2}{*}{ P-value ${ }^{a}$} \\
\hline & & Positive, n (\%) & Negative, n (\%) & \\
\hline Sex & & & & 0.987 \\
\hline Male & 234 & $42(17.9)$ & $192(82.1)$ & \\
\hline Female & 161 & $29(18.0)$ & $132(82.0)$ & \\
\hline Age, years & & & & 0.808 \\
\hline$\leq 50$ & 211 & $37(17.5)$ & $174(82.5)$ & \\
\hline$>50$ & 184 & $34(18.5)$ & $150(81.5)$ & \\
\hline KPS & & & & 0.254 \\
\hline$\leq 60$ & 79 & $15(19.0)$ & $64(81.0)$ & \\
\hline$>60$ & 205 & $31(15.1)$ & $174(84.9)$ & \\
\hline NA & 111 & $25(22.5)$ & $86(77.5)$ & \\
\hline Tumor grade & & & & $<0.001$ \\
\hline II & 60 & $0(0.0)$ & $60(100.0)$ & \\
\hline III & 66 & $6(9.1)$ & $60(90.9)$ & \\
\hline IV & 269 & $65(24.2)$ & $204(75.8)$ & \\
\hline Histology & & & & $<0.001$ \\
\hline A & 36 & $0(0.0)$ & $36(100.0)$ & \\
\hline $\mathrm{O}$ & 21 & $0(0.0)$ & $21(100.0)$ & \\
\hline AA & 40 & $4(10.0)$ & $36(90.0)$ & \\
\hline $\mathrm{AO}$ & 24 & $2(8.3)$ & $22(91.7)$ & \\
\hline GBM & 269 & $65(24.2)$ & $204(75.8)$ & \\
\hline Other & 5 & $0(0.0)$ & $5(100.0)$ & \\
\hline
\end{tabular}

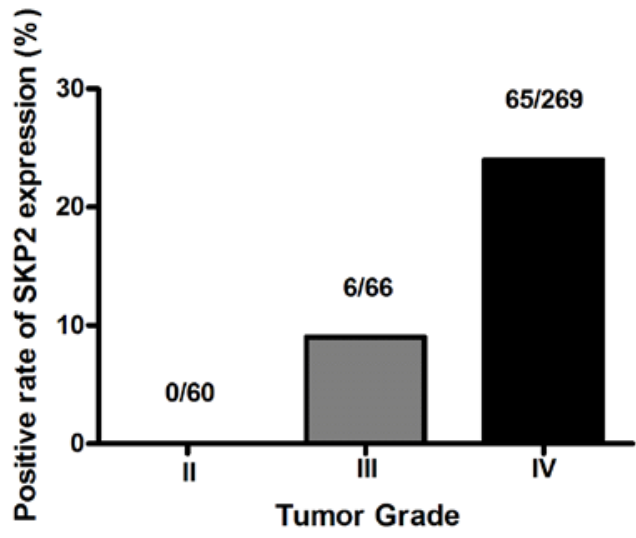

Figure 2. The positive cell rates of SKP2 expression in II, III and IV grade gliomas were $0,9.1$ and $24.2 \%$, respectively. The tumor grading system referred to is the 2016 WHO classification system. SKP2, S phase kinase-associated protein 2.

mutation in gliomas (28). In the present study, it was observed that patients with IDH1 mutation showed favorable prognosis ( $\mathrm{P}=0.048$; Fig. S2A). Furthermore, the TERT promoter mutation is associated with the OS of patients with GBM (P=0.004; Fig. S2B). When SKP2, IDH1 mutation and TERT promoter $(\mathrm{p})$ mutation factors are combined, patients having 3 protective factors (SKP2-/IDH1 ${ }^{+} / \mathrm{TERTp}^{-}$) had the longest OS time in all enrolled gliomas compared with the patients having 2 protective factors $\left(\mathrm{SKP}^{-} / \mathrm{IDH}^{+} / \mathrm{TERTp}^{+}\right.$; $\mathrm{SKP}^{+} / \mathrm{IDH}^{+} / \mathrm{TERTp}^{-}$; SKP2 $/ \mathrm{IDH}^{-} / \mathrm{TERTp}^{-}$) (median OS time, 20.1 months; $\mathrm{P}=0.010$; Fig. 5A). Patients with $<=1$ protective factor $\left(\mathrm{SKP}^{-} / \mathrm{IDH}^{-} / \mathrm{TERTp}^{+}\right.$; $\mathrm{SKP}^{+} / \mathrm{IDH}^{+} / \mathrm{TERTp}^{+}$; $\mathrm{SKP}^{+} / \mathrm{IDH}^{-} / \mathrm{TERTp}^{-}$; or SKP2 ${ }^{+} / \mathrm{IDH}^{-} / \mathrm{TERTp}^{+}$) had the least favorable prognosis (median OS time, 12.2 months; $\mathrm{P}<0.001$; Fig. 5A). Furthermore, patients with GBM demonstrated similar results with median OS times not attained, 18.5 and 12.2 months (those with 3,2 or $<=1$ protective factors, respectively; $\mathrm{P}<0.001$; Fig. $5 \mathrm{~B})$.

\section{Discussion}

SKP2 plays an essential role in cellular biological processes in vitro (7). The present study, demonstrated that SKP2 protein is upregulated in glioma tissues and its expression is strongly associated with tumor grade and poor prognosis. In addition, SKP2 expression was associated with $\mathrm{p}-\mathrm{Rb}$ and 
A
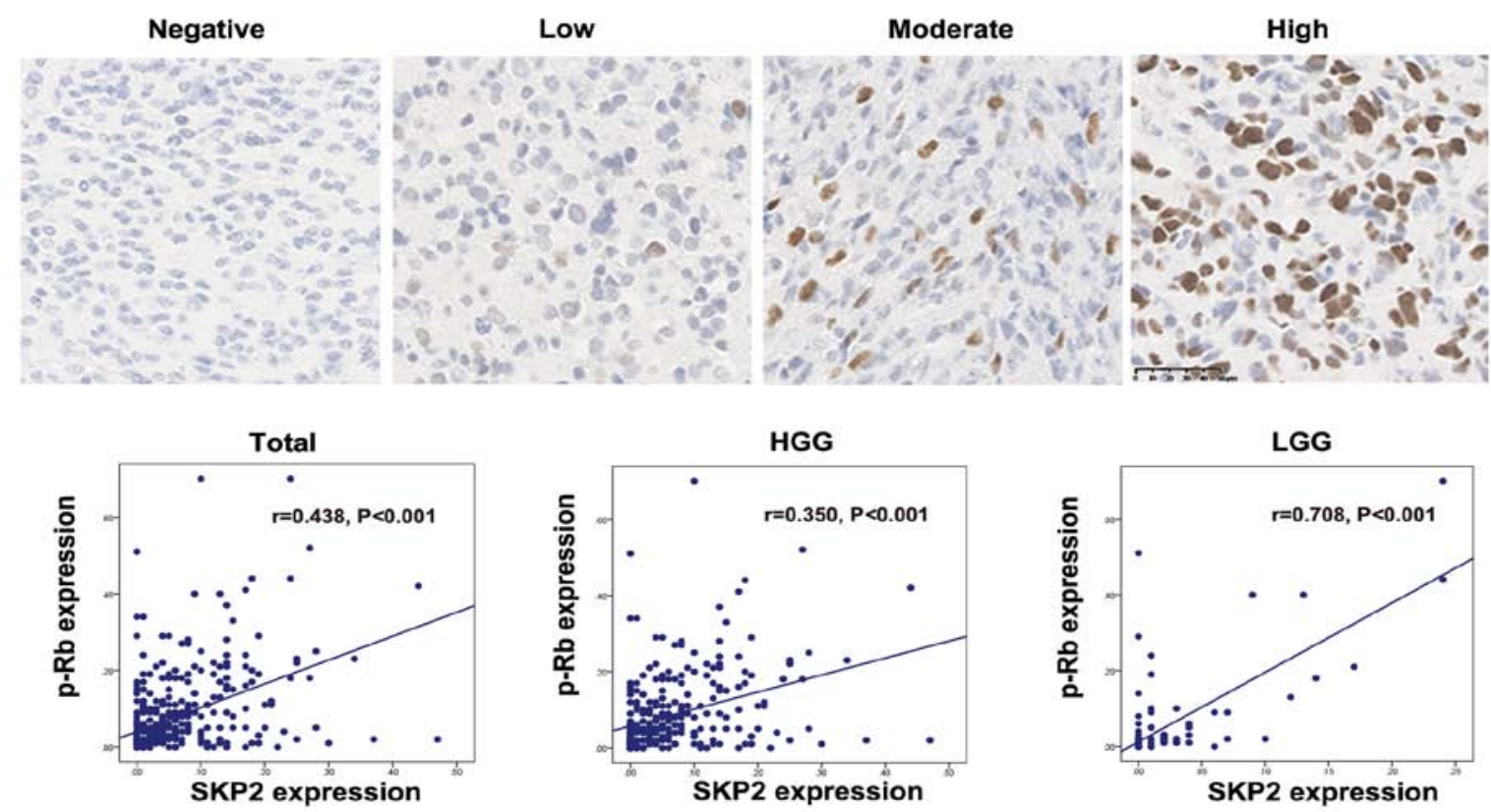

c
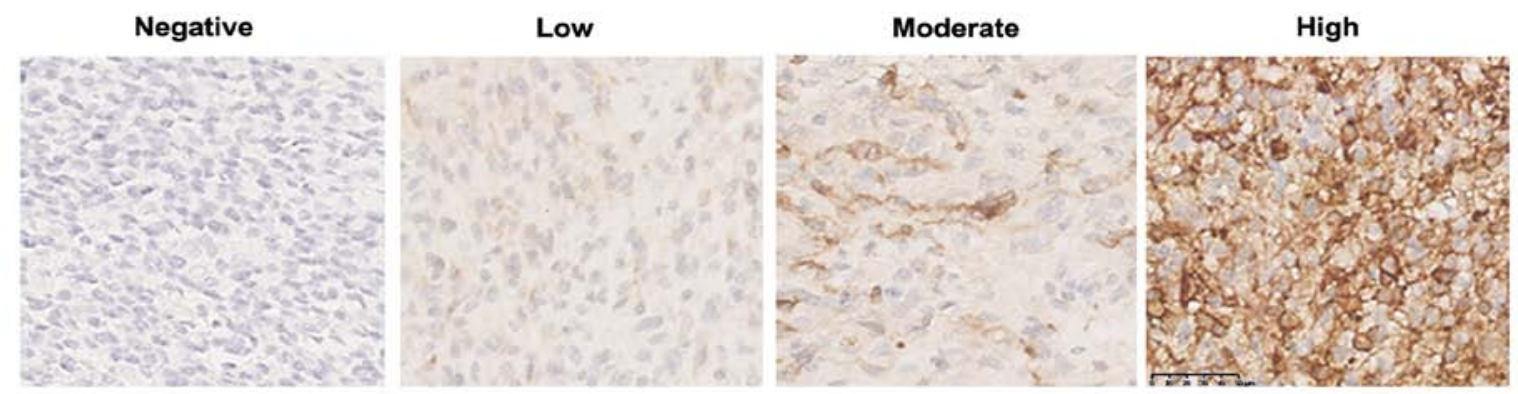

D

Total

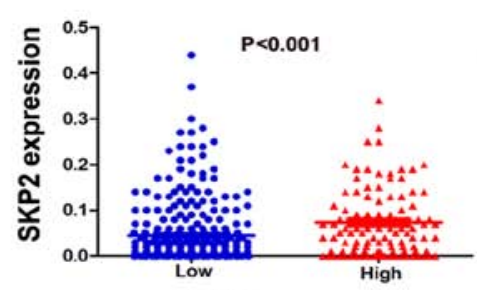

EGFR expression
HGG

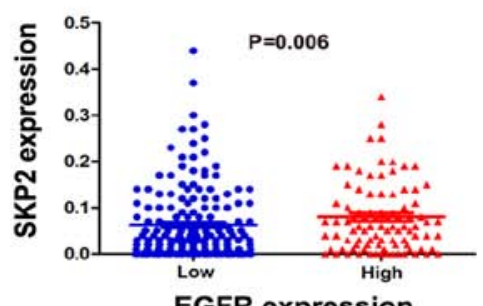

EGFR expression

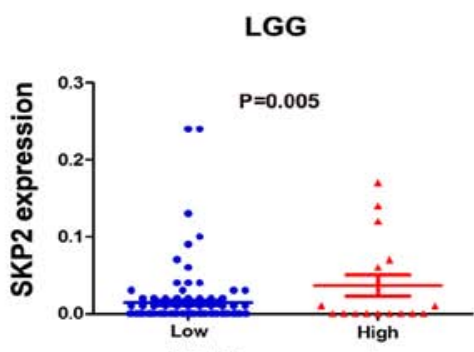

EGFR expression

Figure 3. Correlation of SKP2 expression with other biomarkers in 395 glioma tissues. (A) Blue and brown staining represents negative and positive expression of p-Rb, respectively. (B) According to linear correlation analysis, SKP2 was positively correlated with p-Rb in both HGG and LGG groups. ' $r$ ' means the coefficient of correlation. (C) Blue and brown staining represents negative and positive expression of EGFR, respectively. (D) Positive immunostaining was regarded as high EGFR expression. And no immunostaining was regarded as low EGFR expression. Mann-Whitney U test was used to evaluate the association between SKP2 and EGFR. SKP2, S phase kinase-associated protein 2; EGFR, epidermal growth factor receptor; $\mathrm{p}$-Rb, phosphorylated retinoblastoma protein; HGG, high-grade glioma; LGG, low-grade glioma.

EGFR expression, which suggests that SKP2 may be involved in promoting growth and proliferation in GBM cells. Patients with GBMs were stratified into 3 groups using a combination of SKP2 expression, TERT promoter and IDH1 status, with the longest survival time observed in the patients who were SKP2 $/$ IDH1 ${ }^{+} / \mathrm{TERTp}^{-}$.

Cell cycle-associated proteins have become increasingly important in the understanding of the pathogenesis and prognosis of gliomas (29). SKP2 serves an oncogenic role in cancer cells, primarily through regulating the cell cycle (9). It promotes the transition of the cell cycle from $G_{1}$ to $\mathrm{S}$ phase and subsequently enhances cell proliferation and tumor growth $(30,31)$. An in vitro study reported that SKP2 protein is selectively expressed in a subset of proliferating human breast cancer cells (32). Lu et al (33) demonstrated that SKP2 expression is associated with the histological grade and tumor size in human hepatocellular carcinoma. Furthermore, Elsherif et al (34) also demonstrated that SKP2 proteins serve as a predictor of grade and stage in non-muscle invasive urothelial bladder carcinoma. Similar to the previous studies on other tumor types, the present study observed that SKP2 was upregulated in gliomas and was an independent prognostic 
A

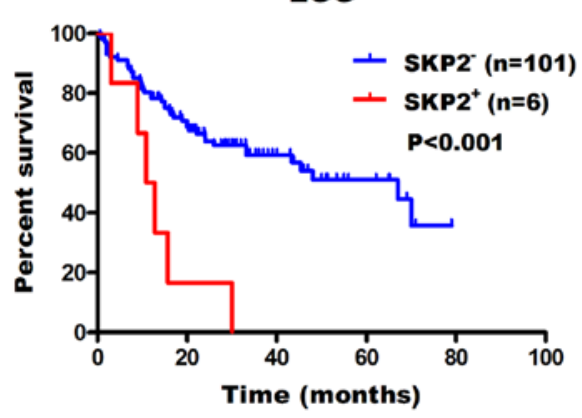

C

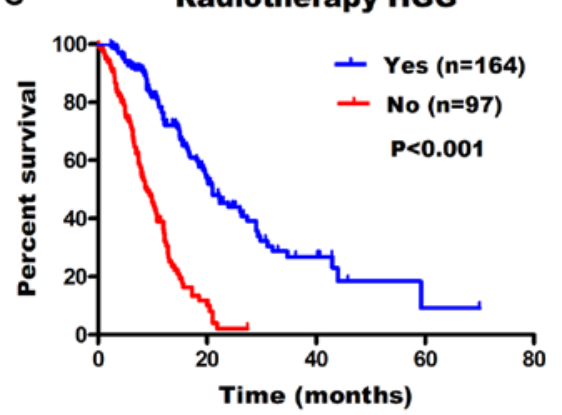

E

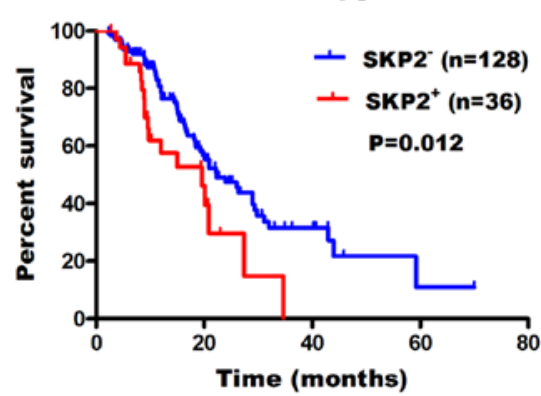

B

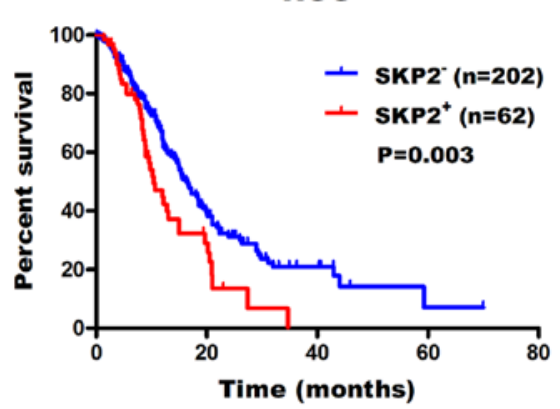

D Chemotherapy HGG

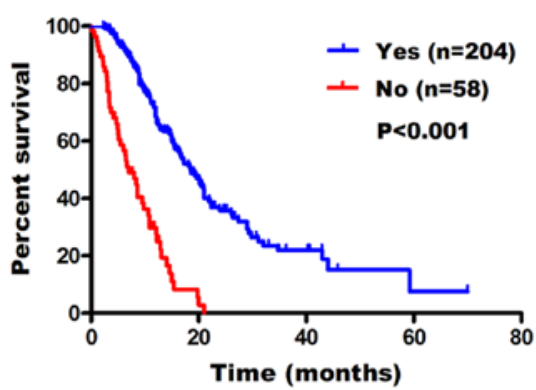

$\mathbf{F}$

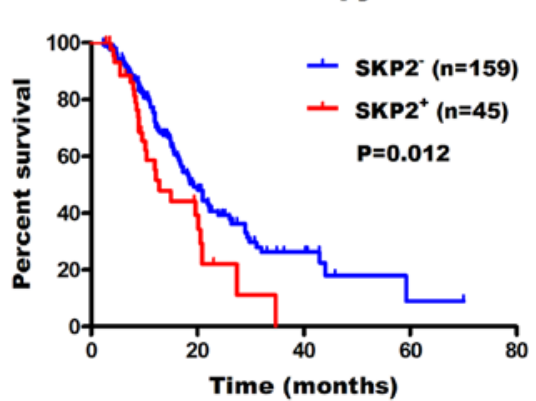

Figure 4. Prognostic value of SKP2 and therapy. SKP2 expression over $>10 \%$ was defined as high SKP2 group (SKP2 $2^{+}$, and expression $<10 \%$ was defined as low SKP2 group (SKP2-). 'Yes', patients received radiotherapy or chemotherapy. 'No', patients have not received radiotherapy or chemotherapy. (A) High SKP2 $\left(\mathrm{SKP} 2^{+}\right)$expression indicates shorter survival time in LGG $(\mathrm{P}<0.001)$. (B) High SKP2 (SKP2+) expression indicates shorter survival time in HGG $(\mathrm{P}=0.003)$. (C) Radiotherapy significantly improves patient survival in $\mathrm{HGG}(\mathrm{P}<0.001)$. (D) Chemotherapy significantly improves patient survival in $\mathrm{HGG}$ ( $\mathrm{P}<0.001)$. (E) Patients with low SKP2 (SKP2-) expression are more sensitive to radiotherapy compared with those that have high SKP2 expression (P=0.012). (F) Patients with chemotherapy observed similar results $(\mathrm{P}=0.012)$. SKP2, S phase kinase-associated protein 2; LGG, low-grade glioma; HGG, high-grade glioma.

factor in GBM. This coincides with the high proliferation activity profile of glioma cells, particularly in GBM (35).

Certain chemical agents including curcumin, paeoniflorin, physcion 8-O- $\beta$-glucopyranoside, escitalopram oxalate and butylidenephthalide have also been revealed to regulate the cell cycle in glioma cells through modulating SKP2 expression (14,15,36-38). Therefore, these agents may have potential for clinical use in the treatment of gliomas with high SKP2 expression. Davidovich et al (39) reported that patients with breast cancer that had high SKP2 expression exhibited a poor response towards preoperative doxorubicin-based chemotherapy. High SKP2 expression in human lung cancer cells caused paclitaxel resistance by downregulating p27 expression (12). Treatment with a combination of small molecule SKP2 inhibitors and paclitaxel conferred a favorable prognosis in patients with lung cancer (12). A combination of SKP2 downregulation and these chemotherapeutic agents may have synergistic implications on tumor control (5). In the present study, high expression of SKP2 resulted in insensitivity to radio- and chemotherapy in glioblastoma. Therefore, SKP2 targeted therapy is required for patients with glioblastoma with high SKP2 expression in addition to standard therapy.

As an important cell cycle regulating protein, $\mathrm{p}-\mathrm{Rb}$ serves pivotal roles in tumorigenesis (24). Xu et al (40) used egg antigen p40 of schistosoma japonicum (Sjp40) to trigger the expression of p27 in LX-2 cells and observed that both SKP2 and $\mathrm{p}-\mathrm{Rb}$ were downregulated. Overexpression of SKP2 reversed p27 protein levels and partially reversed the inhibition of $\mathrm{p}-\mathrm{Rb}$ expression in Sjp40 treated LX2 cells (40). In the present study, consistent alteration of SKP2 with p-Rb was observed in glioma tissues.

EGFR is the target of antineoplastic drugs, such as gefitinib (25). Agents targeting EGFR may promote the 
Table II. Univariate and multivariate regression analyses of overall survival in patients with GBM.

GBM

\begin{tabular}{|c|c|c|c|c|c|c|}
\hline \multirow[b]{3}{*}{ Variables } & \multirow{2}{*}{\multicolumn{2}{|c|}{ Univariate }} & & & & \\
\hline & & & \multirow[b]{2}{*}{ P-value } & \multicolumn{2}{|c|}{ Multivariate } & \multirow[b]{2}{*}{ P-value } \\
\hline & HR & $95 \% \mathrm{CI}$ & & HR & $95 \% \mathrm{CI}$ & \\
\hline Sex, male vs. female & 0.962 & $0.698-1.327$ & 0.814 & & & \\
\hline Age, $\leq 50$ vs. $>50$ years & 1.153 & $0.839-1.583$ & 0.381 & & & \\
\hline KPS,$\leq 60$ vs. $>60$ & 0.530 & $0.355-0.791$ & 0.002 & 0.580 & $0.364-0.923$ & 0.021 \\
\hline Chemo/radiotherapy, no vs. yes & 0.253 & $0.179-0.357$ & $<0.001$ & 0.246 & $0.150-0.403$ & $<0.001$ \\
\hline TERT promoter mutation, no vs. yes & 1.630 & $1.157-2.297$ & 0.005 & 1.440 & $0.931-2.230$ & 0.102 \\
\hline IDH1 mutation, no vs. yes & 0.682 & 0.466-0.999 & 0.049 & 0.502 & $0.292-0.864$ & 0.013 \\
\hline SKP2 expression, no vs. yes & 1.715 & $1.196-2.457$ & 0.003 & 1.767 & $1.045-2.988$ & 0.034 \\
\hline
\end{tabular}

$\mathrm{P}<0.05$ was regarded as statistically significant. GBM, glioblastoma; SKP2, S phase kinase-associated protein 2; IDH 1, isocitrate dehydrogenase 1; TERTp, telomerase reverse transcriptase promoter; KPS, Karnofsky Performance Status; HR, hazard ratio; CI, confidence interval.

A

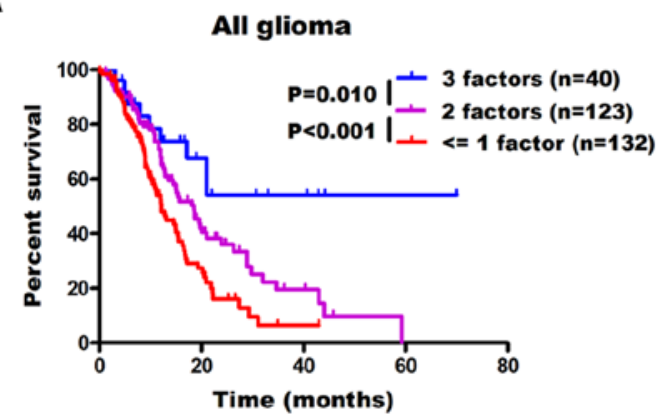

B

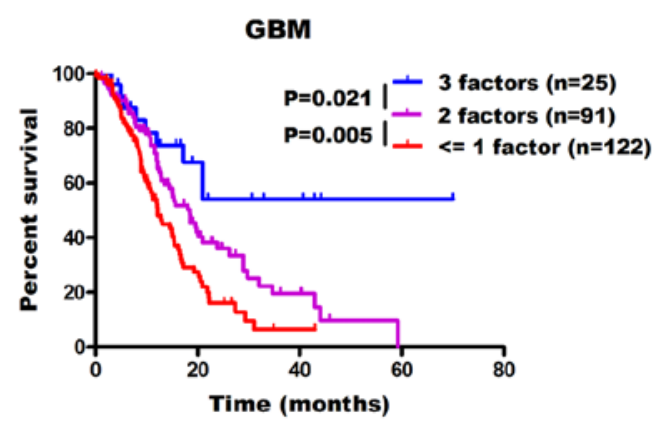

Figure 5. Using SKP2 expression, IDH1 mutation and TERT promoter status for subgroup stratification of patients with (A) all and (B) GBM. Patients with glioma were split into 3 groups according to the number of protective factors they had, including low SKP2 expression, IDH1 mutation and TERT promoter

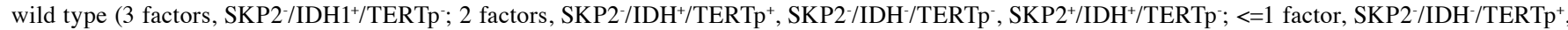
$\mathrm{SKP}^{+} / \mathrm{IDH}^{+} / \mathrm{TERTp}^{+}, \mathrm{SKP}^{+} / \mathrm{IDH}^{-} / \mathrm{TERTp}^{-}, \mathrm{SKP}^{+} / \mathrm{IDH}^{-} / \mathrm{TERTp}^{+}$. GBM, glioblastoma; SKP2, S phase kinase-associated protein 2; IDH 1, isocitrate dehydrogenase 1; TERTp, telomerase reverse transcriptase promoter.

downregulation of SKP2 (25). Tan et al (41) also revealed that upregulated EGFR expression activates the AKT/SKP2 pathway and increases SKP2 expression. In the present study, $38.5 \%$ of patients with HGG who had high SKP2 expression also had EGFR expression. Further studies should investigate the association between these molecules in larger samples and explore whether and how they could be used for the classification of human gliomas.

An increasing number of studies have demonstrated the clinical value of the TERT promoter and IDH1 mutations for classifying patients into molecular subtypes in glioma $(42,43)$. The present study used SKP2 expression combined with TERT promoter and IDH1 mutations to classify patients into subgroups. The results revealed that patients with low SKP2 expression, having an IDH1 mutation and wild-type TERT promoter (SKP2-/IDH1 ${ }^{+} / \mathrm{TERTp}^{-}$) had the best prognosis in all gliomas and GBMs, compared with the other subtypes. A previous studies have demonstrated that IDH1 mutation causes cell cycle arrest in $\mathrm{G}_{1}$ via downregulation of cyclin-dependent kinase 1 expression (44). IDH1 mutation upregulates p21 expression via sterol regulatory element-binding protein 1, inhibits phosphorylation of $\mathrm{Rb}$ and promotes the progression of the cell cycle from $G_{1}$ to $S$ phase (45). The involvement of telomerase in regulating the cell cycle is primarily dependent on the progression of S phase (46). TERT promoter mutation can increase the telomerase reverse transcriptase expression and telomerase activity (47). Thus, SKP $2 /$ IDH $1^{+} /$TERTp $^{-}$ glioma cells in the present study tend to stay in $\mathrm{G}_{1}$ phase and grow slowly. This helps the patient survive longer compared with the other subtypes.

The present study has some limitations. As a retrospective study, the number of cases varied significantly among subgroups, and it was a single-center study. Additionally, the detection method used to determine SKP2 expression was restricted to IHC. New techniques may be required to confirm the results. Finally, the molecular mechanisms of SKP2 have not been explored in the present study.

In conclusion, the present study demonstrated a high expression of SKP2 protein in glioma tissues, and particularly in GBMs. High SKP2 expression is associated with a shorter 
survival time of patients with GBM and is an independent prognostic factor. Gliomas (including GBMs) with low SKP2 expression, wild-type TERT promoter and IDH1 mutation have the longest OS time compared with the other subtypes.

\section{Acknowledgements}

Not applicable.

\section{Funding}

The present study was supported by grants from the CAMS Innovation Fund for Medical Sciences (grant no. 2017-I2M-1-005) and the China Postdoctoral Science Foundation (grant no. 2019M650570).

\section{Availability of data and materials}

The datasets used and/or analyzed during the present study are available from the corresponding author upon reasonable request.

\section{Authors' contributions}

JHW, HQC and MRW designed the experiments and revised the manuscript. ZJC and HQC analyzed the data and wrote the manuscript. MJZ, YZ, JH and QY contributed to the acquisition, analysis and interpretation of data. JJH designed the experiments and analyzed the data. All authors read and approved the final manuscript.

\section{Ethics approval and consent to participate}

The present study was approved by the Ethics Committee of the National Cancer Center/Cancer Hospital, Chinese Academy of Medical Sciences and Peking Union Medical College (Beijing, China; approval no. NCC2014G-12). Patients who participated in this research had complete clinical data. Signed informed consent was obtained from patients or their guardians.

\section{Patient consent for publication}

Not applicable.

\section{Competing interests}

The authors declare that they have no competing interests.

\section{References}

1. Ostrom QT, Gittleman H, Xu J, Kromer C, Wolinsky Y, Kruchko C and Barnholtz-Sloan JS: CBTRUS statistical report: Primary brain and other central nervous system tumors diagnosed in the United states in 2009-2013. Neuro Oncol 18 (Suppl 5): v1-v75, 2016.

2. Louis DN, Perry A, Reifenberger G, von Deimling A, Figarella-Branger D, Cavenee WK, Ohgaki H, Wiestler OD, Kleihues P and Ellison DW: The 2016 world health organization classification of tumors of the central nervous system: A summary. Acta Neuropathol 131: 803-820, 2016.

3. Jan CI, Tsai WC, Harn HJ, Shyu WC, Liu MC, Lu HM, Chiu SC and Cho DY: Predictors of response to autologous dendritic cell therapy in glioblastoma multiforme. Front Immunol 9: 727, 2018.
4. López GY, Van Ziffle J, Onodera C, Grenert JP, Yeh I, Bastian BC Clarke J, Oberheim Bush NA, Taylor J, Chang S, et al: The genetic landscape of gliomas arising after therapeutic radiation. Acta Neuropathol 137: 139-150, 2019.

5. Gong J, Zhou Y, Liu D and Huo J: F-box proteins involved in cancer-associated drug resistance. Oncol Lett 15: 8891-8900, 2018.

6. Wang Z, Liu P, Inuzuka H and Wei W: Roles of F-box proteins in cancer. Nat Rev Cancer 14: 233-247, 2014.

7. Zhang Y, Zvi YS, Batko B, Zaphiros N, O'Donnell EF, Wang J, Sato K, Yang R, Geller DS, Koirala P, et al: Down-regulation of Skp2 expression inhibits invasion and lung metastasis in osteosarcoma. Sci Rep 8: 14294, 2018.

8. Lee SH and McCormick F: Downregulation of Skp2 and p27/Kip1 synergistically induces apoptosis in T98G glioblastoma cells. J Mol Med (Berl) 83: 296-307, 2005.

9. Ding L, Li R, Sun R, Zhou Y, Zhou Y, Han X, Cui Y, Wang W, Lv Q and Bai J: S-phase kinase-associated protein 2 promotes cell growth and motility in osteosarcoma cells. Cell Cycle 16: 1547-1555, 2017.

10. Sonoda H, Inoue H, Ogawa K, Utsunomiya T, Masuda TA and Mori M: Significance of skp2 expression in primary breast cancer. Clin Cancer Res 12: 1215-1220, 2006.

11. Geng Q, Liu J, Gong Z, Chen S, Chen S, Li X, Lu Y, Zhu X, Lin HK and Xu D: Phosphorylation by mTORC1 stablizes Skp2 and regulates its oncogenic function in gastric cancer. Mol Cancer 16: 83, 2017.

12. Huang T, Yang L, Wang G, Ding G, Peng B, Wen Y and Wang Z: Inhibition of Skp2 sensitizes lung cancer cells to paclitaxel. Onco Targets Ther 10: 439-446, 2017.

13. Totary-Jain H, Sanoudou D, Dautriche CN, Schneller H, Zambrana L and Marks AR: Rapamycin resistance is linked to defective regulation of Skp2. Cancer Res 72: 1836-1843, 2012.

14. Li W, Li F, Zhu Y and Song D: Physcion 8-O- $\beta$-glucopyranosideregulates cell cycle, apoptosis, and invasion in glioblastoma cells through modulating Skp2. Biomed Pharmacother 95: 1129-1138, 2017.

15. Ouyang J, Xu H, Li M, Dai X, Fu F, Zhang X and Lan Q: Paeoniflorin exerts antitumor effects by inactivating $\mathrm{S}$ phase kinase-associated protein 2 in glioma cells. Oncol Rep 39: 1052-1062, 2018.

16. Schiffer D, Cavalla P, Fiano V, Ghimenti C and Piva R: Inverse relationship between p27/Kip.1 and the F-box protein Skp2 in human astrocytic gliomas by immunohistochemistry and Western blot. Neurosci Lett 328: 125-128, 2002.

17. Saigusa K, Hashimoto N, Tsuda H, Yokoi S, Maruno M, Yoshimine T, Aoyagi M, Ohno K, Imoto I and Inazawa J: Overexpressed Skp2 within 5p amplification detected by array-based comparative genomic hybridization is associated with poor prognosis of glioblastomas. Cancer Sci 96: 676-683, 2005.

18. Reifenberger G, Wirsching HG, Knobbe-Thomsen CB and Weller M: Advances in the molecular genetics of gliomas-implications for classification and therapy. Nat Rev Clin Oncol 14: 434-452, 2017.

19. Cohen AL, Holmen SL and Colman H: IDH1 and IDH2 mutations in gliomas. Curr Neurol Neurosci Rep 13: 345, 2013.

20. Nonoguchi N, Ohta T, Oh JE, Kim YH, Kleihues P and Ohgaki H: TERT promoter mutations in primary and secondary glioblastomas. Acta Neuropathol 126: 931-937, 2013.

21. Stone JG, Siedlak SL, Tabaton M, Hirano A, Castellani RJ, Santocanale C, Perry G, Smith MA, Zhu X and Lee HG: The cell cycle regulator phosphorylated retinoblastoma protein is associated with tau pathology in several tauopathies. J Neuropathol Exp Neurol 70: 578-587, 2011.

22. Thwaites MJ, Cecchini MJ and Dick FA: Analyzing RB and E2F during the G1-S transition. Methods Mol Biol 1170: 449-461, 2014.

23. Saadeh FS, Mahfouz R and Assi HI: EGFR as a clinical marker in glioblastomas and other gliomas. Int J Biol Markers 33: 22-32, 2018

24. Dick FA and Rubin SM: Molecular mechanisms underlying RB protein function. Nat Rev Mol Cell Biol 14: 297-306, 2013.

25. Shintani S, Li C, Mihara M, Yano J, Terakado N, Nakashiro K and Hamakawa H: Gefitinib ('Iressa', ZD1839), an epidermal growth factor receptor tyrosine kinase inhibitor, up-regulates p27KIP1 and induces G1 arrest in oral squamous cell carcinoma cell lines. Oral Oncol 40: 43-51, 2004.

26. Feng YB, Lin DC, Shi ZZ, Wang XC, Shen XM, Zhang Y, Du XL, Luo ML, Xu X, Han YL, et al: Overexpression of PLK1 is associated with poor survival by inhibiting apoptosis via enhancement of survivin level in esophageal squamous cell carcinoma. Int $\mathrm{J}$ Cancer 124: 578-588, 2009. 
27. Faulkner C, Palmer A, Williams H, Wragg C, Haynes HR, White P, DeSouza RM, Williams M, Hopkins K and Kurian KM: EGFR and EGFRvIII analysis in glioblastoma as therapeutic biomarkers. Br J Neurosurg 29: 23-29, 2014.

28. Cai HQ, Wang PF, Zhang HP, Cheng ZJ, Li SW, He J, Zhang Y, Hao JJ, Wang MR, Yan CX and Wan JH: Phosphorylated Hsp27 is mutually exclusive with ATRX loss and the IDH1(R132H) mutation and may predict better prognosis among glioblastomas without the IDH1 mutation and ATRX loss. J Clin Pathol 71: 702-707, 2018.

29. Soni D, King JA, Kaye AH and Hovens CM: Genetics of glioblastoma multiforme: Mitogenic signaling and cell cycle pathways converge. J Clin Neurosci 12: 1-5, 2005.

30. Hanahan D and Weinberg RA: Hallmarks of cancer: The next generation. Cell 144: 646-674, 2011.

31. Nakayama KI, Hatakeyama S and Nakayama K: Regulation of the cell cycle at the G1-S transition by proteolysis of cyclin $\mathrm{E}$ and p27Kip1. Biochem Biophys Res Commun 282: 853-860, 2001.

32. Signoretti S, Di Marcotullio L, Richardson A, Ramaswamy S, Isaac B, Rue M, Monti F, Loda M and Pagano M: Oncogenic role of the ubiquitin ligase subunit Skp2 in human breast cancer. J Clin Invest 110: 633-641, 2002.

33. Lu M, Ma J, Xue W, Cheng C, Wang Y, Zhao Y, Ke Q, Liu H, Liu Y, Li P, et al: The expression and prognosis of FOXO3a and Skp2 in human hepatocellular carcinoma. Pathol Oncol Res 15: 679-687, 2009.

34. Elsherif E, Elbaky TA, Elserafy F, Elkady N, Dawood M, Gaber MA, Badawy A and Gharabawy ME: $\beta$-catenin and SKP2 proteins as predictors of grade and stage of non-muscle invasive urothelial bladder carcinoma. Chin Clin Oncol 5: 6, 2016.

35. Raucher D, Dragojevic S and Ryu J: Macromolecular drug carriers for targeted glioblastoma therapy: Preclinical studies, challenges, and future perspectives. Front Oncol 8: 624, 2018.

36. Wang L, Ye X, Cai X, Su J, Ma R, Yin X, Zhou X, Li H and Wang Z: Curcumin suppresses cell growth and invasion and induces apoptosis by down-regulation of Skp2 pathway in glioma cells. Oncotarget 6: 18027-18037, 2015.

37. Chen VC, Hsieh YH, Chen LJ, Hsu TC and Tzang BS: Escitalopram oxalate induces apoptosis in U-87MG cells and autophagy in GBM8401 cells. J Cell Mol Med 22: 1167-1178, 2018.

38. Huang MH, Lin SZ, Lin PC, Chiou TW, Harn YW, Ho LI, Chan TM, Chou CW, Chuang CH, Su HL and Harn HJ: Brain tumor senescence might be mediated by downregulation of S-phase kinase-associated protein 2 via butylidenephthalide leading to decreased cell viability. Tumour Biol 35: 4875-4884, 2014.
39. Davidovich S, Ben-Izhak O, Shapira M, Futerman B and Hershko DD: Over-Expression of Skp2 is associated with resistance to preoperative doxorubicin-based chemotherapy in primary breast cancer. Breast Cancer Res 10: R63, 2008.

40. Xu T, Chen J, Zhu D, Chen L, Wang J, Sun X, Hu B and Duan Y: Egg antigen p40 of schistosoma japonicum promotes senescence in activated hepatic stellate cells via SKP2/P27 signaling pathway. Sci Rep 7: 275, 2017.

41. Tan Y, Zhou G, Wang X, Chen W and Gao H: USP18 promotes breast cancer growth by upregulating EGFR and activating the AKT/Skp2 pathway. Int J Oncol 53: 371-383, 2018.

42. Eckel-Passow JE, Lachance DH, Molinaro AM, Walsh KM, Decker PA, Sicotte H, Pekmezci M, Rice T, Kosel ML, Smirnov IV, et al: Glioma groups based on 1p/19q, IDH, and TERT promoter mutations in tumors. N Engl J Med 372: 2499-2508, 2015

43. Diplas BH, He X, Brosnan-Cashman JA, Liu H, Chen LH, Wang Z, Moure CJ, Killela PJ, Loriaux DB, Lipp ES, et al: The genomic landscape of TERT promoter wildtype-IDH wildtype glioblastoma. Nat Commun 9: 2087, 2018.

44. Wang JB, Dong DF, Wang MD and Gao K: IDH1 overexpression induced chemotherapy resistance and IDH1 mutation enhanced chemotherapy sensitivity in Glioma cells in vitro and in vivo. Asian Pac J Cancer Prev 15: 427-432, 2014.

45. Miyata S, Urabe M, Gomi A, Nagai M, Yamaguchi T, Tsukahara T, Mizukami H, Kume A, Ozawa K and Watanabe E: An R132H mutation in isocitrate dehydrogenase 1 enhances $\mathrm{p} 21$ expression and inhibits phosphorylation of retinoblastoma protein in glioma cells. Neurol Med Chir (Tokyo) 53: 645-654, 2013.

46. Chien MN, Yang PS, Hsu YC, Liu TP, Lee JJ and Cheng SP: Transcriptome analysis of papillary thyroid cancer harboring telomerase reverse transcriptase promoter mutation. Head Neck 40: 2528-2537, 2018.

47. Jafri MA, Ansari SA, Alqahtani MH and Shay JW: Roles of telomeres and telomerase in cancer, and advances in telomerase-targeted therapies. Genome Med 8: 69, 2016.

(i) (8) This work is licensed under a Creative Commons Attribution-NonCommercial-NoDerivatives 4.0 International (CC BY-NC-ND 4.0) License. 